\title{
Endothelial cell-derived CCL15 mediates the transmigration of fibrocytes through the CCL15-CCR1 axis in vitro
}

\author{
NAN PANG $^{1 *}$, ZHIXIAO LIN $^{1 *}$, XIAOLIN WANG ${ }^{1}$, LIRONG XU $^{1}$, XIAOLI XU ${ }^{1}$, \\ RONG HUANG ${ }^{1}$, XINGXING LI ${ }^{2}$, XUEYONG LI ${ }^{1}$ and JINQING LI ${ }^{1}$ \\ ${ }^{1}$ Department of Plastic Surgery, Tangdu Hospital, Fourth Military Medical University, Xi'an, Shaanxi 710038; \\ ${ }^{2}$ Department of Prosthodontics, Kunming Medical University, Kunming, Yunnan 650500, P.R. China
}

Received February 1, 2020; Accepted September 8, 2020

DOI: $10.3892 / \mathrm{mmr} .2020 .11610$

\begin{abstract}
Wound healing is a complex physiological process in which fibrocytes serve a vital role. However, the mechanism underlying the recruitment of fibrocytes during wound healing remains largely unknown. The present study aimed to investigate whether endothelial cells are involved in the recruitment of fibrocytes in wound healing. To mimic the in vivo angiogenic process, a co-culture system consisting of endothelial cells and fibrocytes was achieved using a permeable Transwell co-culture system. The expression of chemokines produced by endothelial cells with or without co-culture was then measured using a gene chip. Based on the dataset from chip analysis, chemokine ligand 15 (CCL15) produced by endothelial cells was identified, which likely serves a regulatory role in mediating the transmigration of fibrocytes. Overexpression of CCL15 in endothelial cells or chemokine receptor 1 (CCR1) in fibrocytes promoted the transmigration of fibrocytes, whilst silencing the expression of CCL15 in endothelial cells or that of CCR1 in fibrocytes attenuated the transmigration of fibrocytes. Results from the present study suggested that the CCL15-CCR1 axis between endothelial cells and fibrocytes serves a vital role in mediating the recruitment of fibrocytes during wound healing.
\end{abstract}

\section{Introduction}

Wound healing is a complex physiological process. In the clinic setting, enhancing wound healing has long been a goal

Correspondence to: Professor Xueyong Li or Dr Jinqing Li, Department of Plastic Surgery, Tangdu Hospital, Fourth Military Medical University, 569 Xinsi Road, Xi'an, Shaanxi 710038, P.R. China

E-mail: lixueyong641123@163.com

E-mail: lijqfmmu@hotmail.com

*Contributed equally

Key words: human umbilical vein endothelial cell, circulating fibrocyte, chemokine ligand 15 , chemokine receptor 1 , chemotaxis of physicians. Patients with certain conditions are particularly susceptible to chronic, non-healing wounds, such as those of diabetic wounds (1). In addition, tending to chronic and intractable wounds increases the cost of healthcare (2). Therefore, elucidating the mechanism underlying wound healing so that this process is accelerated is currently necessary and urgently sorted.

Circulating fibrocytes (CFs), also termed peripheral blood fibrocytes, are bone-marrow derived mesenchymal stem cells that account for $\sim 1 \%$ of the total number of mononuclear cells in the blood (3). Chen et al (4) previously reported that fibrocytes accelerate wound healing by stimulating cell proliferation, re-epithelialization and angiogenesis in a mouse diabetic model. Notably, a previous study demonstrated that CFs can differentiate into pericytes, which are indispensable components of new blood vessels (5). Additionally, fibrocytes have been documented to induce an angiogenic phenotype in cultured endothelial cells (6) and contribute to the stabilization of newly-formed vessels during angiogenesis (5). Recruitment of fibrocytes into wound areas forms the basis of their functions (4). Thymic stromal lymphopoietin has been reported to function in airway remodeling by promoting $\mathrm{CF}$ recruitment to the lungs in mice subjected to chronic allergen exposure (7). In addition, contribution of chemokines chemokine ligand (CCL)5, CCL11 and CCL24 in the recruitment of fibrocytes to the airway in severe asthma has also been previously demonstrated (8). Aside from asthma, fibrocyte numbers have also been observed to be increased in the circulating blood in patients with necrotizing enterocolitis, where they may be recruited to the inflammatory intestinal tract through the CXC chemokine receptor (CXCR) 4-CXC chemokine ligand (CXCL) 12 axis (9). Platelet-derived growth factor (PDGF)-BB-PDGF receptor- $\beta$ biological axis has also been reported to serve a role in the transmigration of fibrocytes into fibrotic lungs (10). Although the mechanism underlying the transmigration of fibrocytes has been explored in numerous studies, an insufficient number studies investigated the mechanism associated with the transmigration of fibrocytes during the wound healing process. In particular, chemokines that participate in the recruitment of fibrocytes during wound healing remain poorly understood. Based on the data from gene chip analysis (Pang, unpublished data), it was hypothesized that a specific chemokine secreted by endothelial cells in newly-formed blood vessels during wound healing may 
mediate the chemotaxis of fibrocytes towards endothelial cells in the wound through a cognate receptor expressed on fibrocytes.

The present study aimed to explore the role of chemokines secreted by human umbilical vein endothelial cells (HUVECs) in mediating the transmigration of CFs. Gene chip analysis was first applied to measure the expression levels of chemokines in HUVECs that were co-cultured with CFs in a permeable Transwell system or cultured alone. Based on these data from this gene chip, it was hypothesized that some chemokines may be involved in mediating the transmigration of fibrocytes toward HUVECs. Further transmigration assay experiments were performed to identify the functional role of screened chemokines and its corresponding receptor. The present study demonstrated that the chemokine ligand 15 (CCL15) and its receptor chemokine receptor 1 (CCR1) are involved in mediating the recruitment of fibrocytes to HUVECs, which may provide a new target for accelerating angiogenesis, which can in turn accelerate wound healing.

\section{Materials and methods}

Cell isolation and culture. Human CFs were isolated and purified as previously described (11). Briefly, human CFs were isolated from leukapheresis packs (6 donors; male; age range, 20-40 years old) provided by the Xijing Hospital Blood Center (Xi'an, China) using the Histopaque ${ }^{\circledR}-1077$ (cat. no. 10771; Sigma-Aldrich; Merck KGaA) density gradient method. The leukapheresis sample was mixed with PBS (1:1), before the diluted sample was layered over Histopaque ${ }^{\circledR}-1077$ (2:1) and centrifuged at $800 \mathrm{x}$ g for $20 \mathrm{~min}$ at room temperature. The peripheral blood mononuclear cell (PBMC) layer was acquired by gentle aspiration, which was washed with PBS and centrifuged at $1,200 \mathrm{x}$ g for $5 \mathrm{~min}$ at room temperature three times. The PBMC layer was resuspended in DMEM (HyClone; Cytiva) supplemented with $4.0 \mathrm{mM}$ L-glutamine, $4,500 \mathrm{mg} / 1$ glucose, $100 \mathrm{U} / \mathrm{ml}$ penicillin-G and $100 \mathrm{U} / \mathrm{ml}$ streptomycin. PBMCs were then seeded into six-well plates at a density of $1 \times 10^{7}$ cells $/ \mathrm{ml}$ and 24-well plates at a density of $5 \times 10^{5}$ cells $/ \mathrm{ml}$ and cultured at $37^{\circ} \mathrm{C}$ with $5 \% \mathrm{CO}_{2}$. After 7 days of culture, non-adherent cells were removed from the culture dishes and CFs were retained, and the media was replaced. After 14 days of culture, the resultant enriched human fibrocyte populations were $>95 \%$ pure based on collagen I and CD34 immunohistochemical staining, which was performed as described previously (11).

HUVECs were purchased from ScienCell Research Laboratories (cat. no. 8000). HUVECs were cultured in endothelial cell medium supplemented with 5\% FBS (cat. no. 1001; ScienCell Research Laboratories), $4 \mathrm{mM}$ L-glutamine, $100 \mathrm{U} / \mathrm{ml}$ penicillin-G, $100 \mathrm{U} / \mathrm{ml}$ streptomycin and $1 \%$ endothelial cell growth supplement (v/v; cat. no. 1052; ScienCell Research Laboratories) at $37^{\circ} \mathrm{C}$ with $5 \% \mathrm{CO}_{2}$. HUVECs used in the present study were from passages 3-4. The experimental protocol was approved by the Ethics Committee for Experimentation of the Fourth Military Medical University (Shaanxi, China).

Cell co-culture system. Purified CFs and HUVECs were separately cultured in six-well plates for 2 days at $37^{\circ} \mathrm{C}$ with $5 \% \mathrm{CO}_{2}$ prior to co-culture. In the co-culture system, the HUVECs were suspended in endothelial cell medium (ScienCell Research Laboratories, Inc.) at a density $1 \times 10^{5}$ cells $/ \mathrm{ml}$ and added into the lower chamber before the CFs were suspended in DMEM at a density $1 \times 10^{5}$ cells $/ \mathrm{ml}$ and added into the upper Transwell inserts (cat. no. 140640; Thermo Scientific; Thermo Fisher Scientific, Inc.). HUVECs and CFs co-cultured for $24 \mathrm{~h}$ at $37^{\circ} \mathrm{C}$ were then extracted for RNA microarray analysis or used for Transwell migration experiments. HUVECs and CFs that were co-cultured for $48 \mathrm{~h}$ were detached for reverse transcription-quantitative (RT-q) PCR, western blotting and ELISA. Separately cultured HUVECs and CFs in six-well plates were used as the control groups.

Gene chip microassay. Gene chip microarray analysis was performed using HumanWG-6_V3 (Illumina, Inc.) according to the manufacturer's protocol. HUVECs were lysed using TRIzol ${ }^{\circledR}$ (Thermo Fisher Scientific, Inc.) after $24 \mathrm{~h}$ co-culture. Total RNA was then extracted using the TRIzol ${ }^{\circledR}$ reagent, followed by purification and DNase I treatment using a Qiagen RNeasy mini kit (Qiagen $\mathrm{GmbH}$ ) according to the manufacturer's protocol. An Agilent Bioanalyzer (Agilent Technologies $\mathrm{GmbH}$ ) was used for quality control. Biotinylated circular (c)RNA was prepared with the Ambion MessageAmp kit (cat. no. AM1819; Thermo Scientific; Thermo Fisher Scientific, Inc.) for Illumina arrays according to the manufacturer's protocol. Labeled cRNA was hybridized to the probes on the chip and washed. The results were scanned by a SureScan Microarray scanner (cat. no. G4900DA; Agilent Technologies, Inc,) according to the manufacturer's protocol. The data were normalized using quantile normalization by GenomeStudio v2.0 (Illumina, Inc.). All data have been deposited into the Gene Expression Omnibus database and are available with the accession number GSE108626.

$R T-q P C R$. The total RNA of CFs and HUVECs was isolated using a Takara MiniBEST Universal RNA Extraction Kit (cat. no. 9767; Takara Bio, Inc.) according to the manufacturer's protocol. Reverse transcription was performed with PrimeScript ${ }^{\mathrm{TM}}$ RT Master Mix (cat. no. RR036A; Takara Bio, Inc.) according to the manufacturer's protocol. qPCR was subsequently performed using TB Green ${ }^{\mathrm{TM}}$ Premix Ex Taq II (cat. no. RR820A; Takara Bio, Inc.). The sequences of primers used were as follows: CCL15 forward, 5'-CTCTCCTGCCTCATGCTTGT-3' and reverse, 5'-CAGCAGCAAAGTGAAAGCTG-3'; CCL2 forward, 5'-CCCCAGTCACCTGCTGTTAT-3' and reverse, 5'-AGA TCTCCTTGGCCACAATG-3'; CXCL8 forward, 5'-CTG CGCCAACACAGAAATTA-3' and reverse, 5'-TGAATTCTC AGCCCTCTTCAA-3'; CCR1 forward, 5'-CTGGTTGGA AACATCCTGGT-3' and reverse, 5'-GGAAGCGTGAAC AGGAAGAG-3'; CCR3 forward, 5'-TGGCGGTGTTTTTCA TTTTC-3' and reverse, 5'-CCGGCTCTGCTGTGGAT-3'; and GAPDH forward, 5'-AGAAGGCTGGGGCTCATTTG-3' and reverse, 5'-AGGGGCCATCCACAGTCTTC-3'. All primers were obtained from Sangon Biotech Co., Ltd. The PCR amplifications were performed in a CFX Connect Real-Time System (Bio-Rad Laboratories, Inc.) under standard cycling conditions in accordance with the manufacturer's protocol. The following thermocycling conditions were used for the qPCR: 
Initial denaturation at $95^{\circ} \mathrm{C}$ for min; followed by 40 cycles of denaturation at $95^{\circ} \mathrm{C}$ for $15 \mathrm{sec}$, annealing and elongation at $60^{\circ} \mathrm{C}$ for $1 \mathrm{~min}$; then a final extension at $68^{\circ} \mathrm{C}$ for $3 \mathrm{~min}$. Expression levels were quantified using the $2^{-\Delta \Delta \mathrm{Cq}}$ method (12) and normalized to GAPDH. Data were analyzed with CFX Manager software version 2.1 (Bio-Rad Laboratories, Inc.).

ELISAs. The concentrations of chemokines in single culture or co-culture supernatants were detected by ELISAs. CFs and HUVECs were co-cultured as aforementioned. The medium volume in each chamber was $2 \mathrm{ml}$. The media were not changed during this experiment. After a 48-h culture, the culture medium of the HUVECs was collected. The medium samples were centrifuged at $600 \mathrm{x}$ g for $10 \mathrm{~min}$ at room temperature and the supernatant was stored at $-80^{\circ} \mathrm{C}$ for subsequent experiments. The supernatants were diluted 1:2 in serum-free endothelial cell medium before ELISAs were performed with human CCL2/monocyte chemoattractant protein-1 (cat. no. DCP00; R\&D Systems, Inc.), interleukin-8/CXCL8 (cat. no. D8000C; R\&D Systems, Inc.) and macrophage inflammatory protein 5/CCL15 (cat. no. ab100598; Abcam) ELISA kits, according to the manufacturers' protocols. Data were acquired by a Tecan Sunrise ${ }^{\mathrm{TM}}$ microplate absorbance reader (Tecan Group, Ltd.) and normalized based on cell numbers. The experiment was repeated three times.

Chemokine blocking experiment. The polyclonal antibodies used to block the corresponding chemokines were rabbit polyclonal anti-CCL2 (1:10; cat. no. 25542-1-AP; ProteinTech Group, Inc.), rabbit polyclonal anti-CCL15 (1:10; cat. no. ab221040; Abcam) and rabbit polyclonal anti-CXCL8 (1:10; cat. no. ab7747; Abcam). The addition of the antibodies to HUVECs cultured in the co-culture system was performed at $37^{\circ} \mathrm{C}$ for $24 \mathrm{~h}$ with $5 \% \mathrm{CO}_{2}$ prior to further experiments. Control groups were added with the same amount of TE buffer ( $\mathrm{pH}$ 9.0), which is the solvent of these antibodies only.

Western blotting analysis. Western blotting analysis was performed following standard procedures. Briefly, T-PER ${ }^{\mathrm{TM}}$ Tissue Protein Extraction reagent (Thermo Fisher Scientific, Inc.) was used to extract total protein from cells, and the protein content was determined using Bradford assay. Protein samples (30-40 $\mu \mathrm{g} /$ lane) were separated with $8 \%$ SDS-PAGE and transferred onto PVDF membranes. After blocking with 5\% non-fat milk in TBS-0.05\% Tween-20 for $1 \mathrm{~h}$ at room temperature, the PVDF membranes were incubated with anti-CCR1 mouse monoclonal (1:1,000; cat. no. ab129002; Abcam) and anti-actin mouse monoclonal (1:1,000; cat. no. ab11003; Abcam) primary antibodies for $3 \mathrm{~h}$ at $37^{\circ} \mathrm{C}$ and then incubated with a horseradish peroxidase-conjugated rabbit polyclonal anti-mouse IgG-H\&L secondary antibody (1:2,000; cat. no. ab6728; Abcam) for $1 \mathrm{~h}$ at room temperature. Protein bands were visualized with ECL solution and a ChemiDoc XRS+ system (Bio-Rad Laboratories, Inc.). The densities of the specific bands were further analyzed with ImageJ software v1.53a (National Institutes of Health).

CCL15 and CCR1 overexpression. 293T cells (American Type Culture Collection) were kept in DMEM supplemented with $10 \%$ FBS, $1 \%$ glutamine and 1\% P/S antibiotics (Thermo Fisher
Scientific, Inc.) at $37^{\circ} \mathrm{C}$ and $5 \% \mathrm{CO}_{2}$. Human CCL15 and CCR1 genes were cloned into pLenti-CMV-EGFP-3FLAG vectors (OBiO Technology Corp., Ltd.). Using the calcium phosphate transfection protocol (13), 293T cells were transfected with

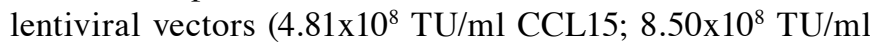
CCR1; $8.41 \times 10^{8} \mathrm{TU} / \mathrm{ml}$ empty vector) together with packaging vectors pMD2-VSVG and pPAX2 (OBiO Technology Corp., Ltd.). Lentiviral particles prepared by transfecting the 293T cells empty lentiviral particle vector together with packaging vectors were used as negative controls. Lentiviruses were harvested $48 \mathrm{~h}$ post transfection and concentrated by ultracentrifugation at $3,000 \mathrm{xg}$ for $2 \mathrm{~h}$ at $4^{\circ} \mathrm{C}$. HUVECs and CFs were then infected with the lentiviral particles at a multiplicity of infection (MOI) of 40 in the presence of $8 \mathrm{mg} / \mathrm{ml}$ polybrene (Sigma-Aldrich; Merck KGaA). The cells were harvested 3-4 days after transfection for subsequent experimentation.

CCL15 and CCR1 knockdown. CCL15 small interfering (si) RNA CCL15-homo-667 (5'-CCAGUAGUUCUGAACAGCU TT-3') was synthesized by Sangon Biotech Co. Ltd. HUVECs were transfected with either si-CCL15 or nonsense siRNA (cat. no. 4404021; Silencer ${ }^{\mathrm{TM}}$ Negative Control No. 1 siRNA; Thermo Fisher Scientific, Inc.) using Lipofectamine ${ }^{\circledR} 2000$ (cat. no. 11668019; Invitrogen; Thermo Fisher Scientific, Inc.) at a concentration of $50 \mathrm{nM}$ according to the manufacturer's protocol. Small harpin (sh)RNA of human CCR1 (5'-CCT ACAATTTGACTATACTT-3') or control shRNA (targeting sequence 5'-TTCTCGAACGTGTCACGT-3') was cloned into the pLKD-CMV-Puro-U6-shRNA vector (OBiO Technology Corp., Ltd.), following which recombinant lentiviruses were generated as aforementioned. CFs were infected with the lentiviral particles at $\mathrm{MOI}=40$ in the presence of $8 \mathrm{mg} / \mathrm{ml}$ polybrene (Sigma-Aldrich; Merck KGaA). The cells were harvested 3-4 days after transfection for subsequent experimentation.

Transwell migration assay. HUVECs were suspended in endothelial cell medium at a density of $1 \times 10^{5}$ cells $/ \mathrm{ml}$ and plated into the lower chamber. The CFs were suspended in DMEM at a density of $1 \times 10^{5}$ cells $/ \mathrm{ml}$ and plated into the upper chamber of the Transwell inserts (cat. no. 140640; Thermo Scientific; Thermo Fisher Scientific, Inc.). After $24 \mathrm{~h}$ of co-culture at $37^{\circ} \mathrm{C}$, the $\mathrm{CFs}$ were fixed with $4 \%$ formaldehyde for $30 \mathrm{~min}$ at room temperature (cat. no. DF0133; Beijing Leagene Biotech Co., Ltd.) and stained with $0.1 \%$ crystal violet (cat. no. C0121; Beyotime Institute of Biotechnology) for $5 \mathrm{~min}$ at room temperature. The number of CFs that migrated through the membrane towards the HUVECs and attached on the underside of the membrane was then counted under a light microscope (magnification, x20) in three randomly selected fields of view per chamber to obtain an average count.

Statistical analysis. All experiments were repeated three times. The data were expressed as the mean \pm SEM. One-way ANOVA was used to compare differences between $>$ two groups of data. Dunnett's post hoc test was performed for the multiple comparison of two groups vs. the control group. Tukey's post hoc test was performed for multiple comparisons of $>$ two groups. An unpaired two-tailed Student's t-test was performed to compare 
A

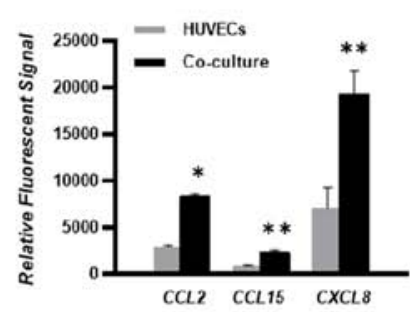

B

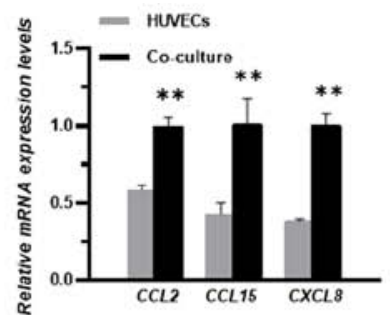

C

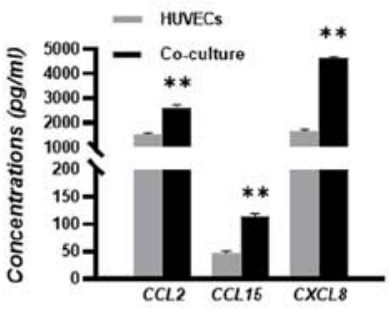

D

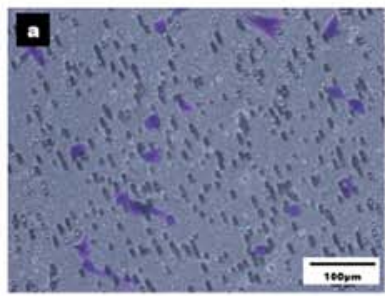

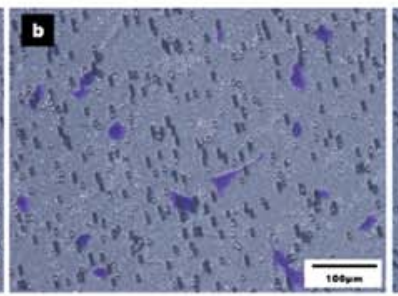
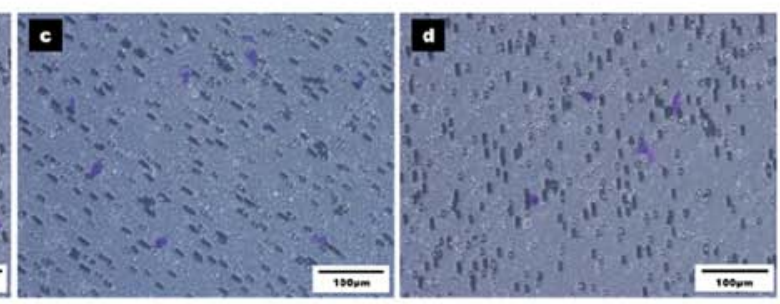

E

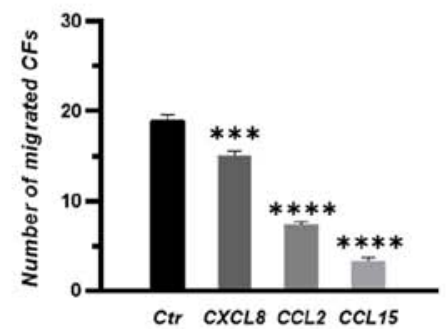

Figure 1.CCL15 is a vital chemokine that mediates the transmigration of CFs toward HUVECs. (A) Expression levels of the three chemokines in mono-cultured and co-cultured HUVECs were measured by gene chip microarray analysis and expressed as relative fluorescent signals. (B) Relative mRNA expression levels of CCL2, CCL15 and CXCL8 in the mono-cultured HUVECs and co-cultured HUVECs were measured by reverse transcription-quantitative PCR (C) Concentrations of CCL2, CCL15 and CXCL8 in the medium of mono-cultured HUVECs and co-cultured HUVECs were measured by ELISA.(D) Migratory CFs were stained and imaged under a microscope. (D-a) Control group, (D-b) CXCL8 blocking group, (D-c) CCL2 blocking group and (D-d) CCL15 blocking group. $\mathrm{n}=3$. Scale bar, $100 \mu \mathrm{m}$. (E) The number of migrated CFs in each group. ${ }^{*} \mathrm{P}<0.05,{ }^{* * *} \mathrm{P}<0.01,{ }^{* * * *} \mathrm{P}<0.001$ and ${ }^{* * * * *} \mathrm{P}<0.0001$ vs. HUVECs (parts A-C) or Ctr (part E). HUVEC, human umbilical vein endothelial cell; $\mathrm{CFs}$, circulating fibrocytes; $\mathrm{Ctr}$, control; $\mathrm{CCL}$, chemokine ligand; CXCL, $\mathrm{CXC}$ chemokine ligand.

the difference between two groups. Statistical analysis was performed using SPSS v19.0 software (IBM Corp.) and figures were plotted using GraphPad Prism 8.0 software (GraphPad Software, Inc.).

\section{Results}

CCL15 is a vital chemokine that mediates the transmigration of CFs toward HUVECs. Previous studies have demonstrated that fibrocytes migrate towards vascular endothelial cells in vitro $(5,6,12,14)$. To study the mechanism underlying the tropism of CFs for HUVECs, a Transwell co-culture system was used to mimic the in vivo communication between endothelial cells and fibrocytes. HUVECs were subjected to gene chip microarray analysis after co-culture with CFs or after culturing alone. The results demonstrated that the expression levels of the chemokines CCL2, CCL15 and CXCL8 were significantly increased in the HUVECs co-cultured with CFs compared with those in HUVECs cultured alone (Fig. 1A and B). Additionally, ELISA results demonstrated that the concentrations of CCL2, CCL15 and CXCL8 were significantly higher in the HUVECs co-cultured with CFs compared with those in HUVECS cultured alone (Fig. 1C).

The transmigration of CFs toward HUVECs was next assessed using Transwell assay. Polyclonal antibodies were used to evaluate the effects of CCL2, CCL15 and CXCL8 on modulating the transmigration of CFs. Notably, a fewer number of migrated CFs was observed following the addition of the CCL15 antibody compared with that following the addition of CCL2 or CXCL8 antibodies (Fig. 1D and E).

CCL15 mediates the transmigration of CFs toward HUVECs in vitro. To investigate the chemotactic role of CCL15, CCL15 in HUVECs were either overexpressed or silenced before transfection efficiency was examined by RT-qPCR and ELISA. The results demonstrated that the expression levels of CCL15 were significantly increased in the CCL15 overexpression group (Fig. 2A and B) and significantly decreased in the si-CCL15 group (Fig. 3A and B) compared with those in negative control (NC) groups. Subsequently, CCL15 overexpression in HUVECs significantly promoted the migration of CFs towards HUVECs (Fig. 2C and D), whilst knocking down CCL15 expression in HUVECs significantly attenuated the transmigration of CFs towards HUVECs in co-culture conditions (Fig. 3C and D).

CCR1 mediates the migration of CFs toward HUVECs in response to CCL15 in vitro. The CCL15-CCR1 chemokine axis has been previously reported to modulate the migration and accumulation of numerous cell types $(15,16)$. Therefore, to investigate whether CFs were recruited by HUVECs through 
A

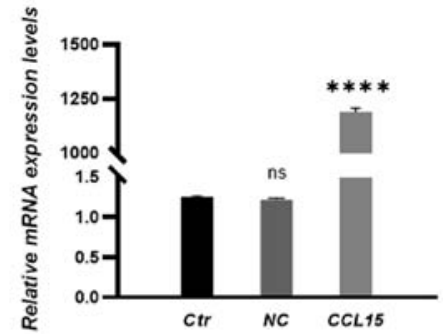

B

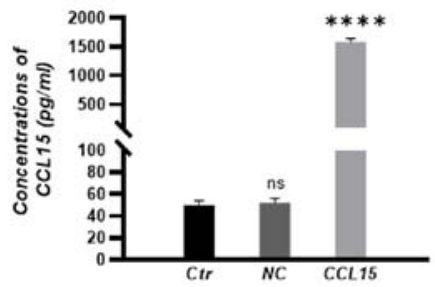

C
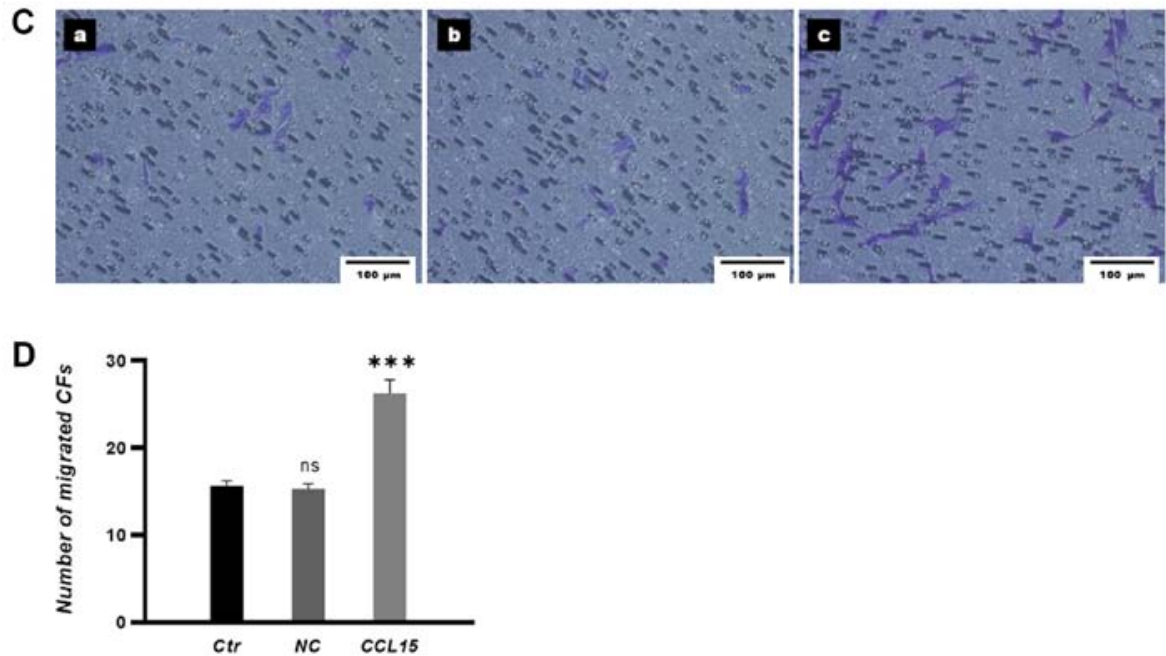

Figure 2. CCL15 overexpression in HUVECs promotes the migration of CFs towards HUVECs. (A) The mRNA expression levels of CCL15 in HUVECs of each group were measured by reverse transcription-quantitative PCR. (B) Concentrations of CCL15 in HUVEC culture supernatants in each group were measured by ELISA. (C) Migrated CFs were stained and imaged under a microscope. (C-a) Control group, (C-b) Negative control group and (C-c) CCL15 group. $\mathrm{n}=3$. Scale bar, $100 \mu \mathrm{m}$. (D) The number of migrated CFs in each group. Untransfected HUVECs were used in the Ctr groups and empty vector transfected HUVECs were used in the NC groups. ${ }^{* * *} \mathrm{P}<0.001$ and ${ }^{* * * * *} \mathrm{P}<0.0001$ vs. NC; ns vs. Ctr. HUVEC, human umbilical vein endothelial cell; $\mathrm{CFs}$, circulating fibrocytes; Ctr, control; NC, negative control; CCL, chemokine ligand; ns, non-significant.

A

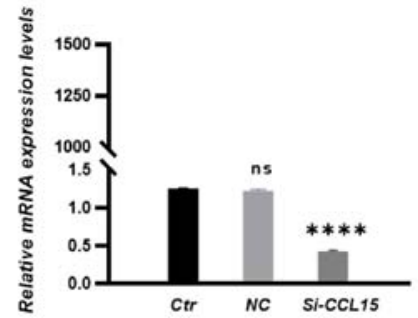

C

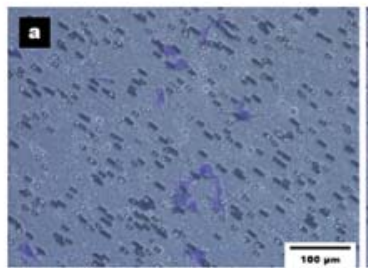

D

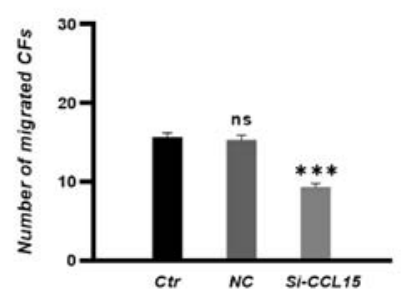

B
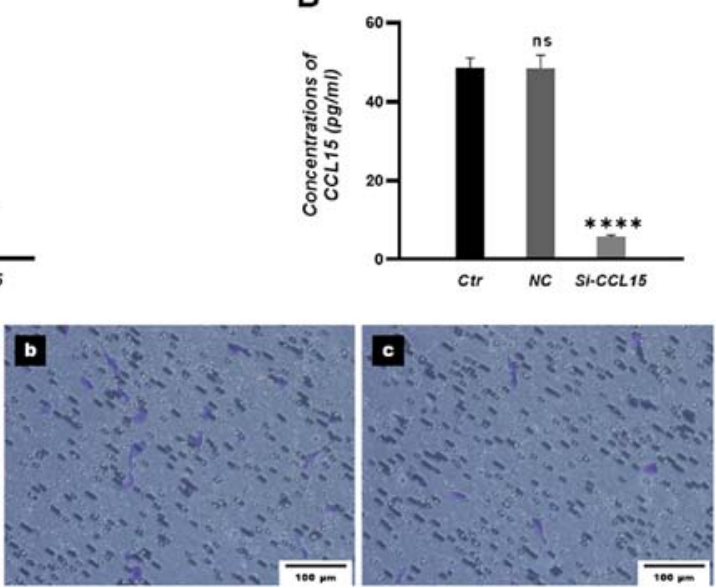

Figure 3. CCL15 knockdown in HUVECs attenuates the migration of CFs towards HUVECs. (A) The mRNA expression levels in HUVECs each group were measured by reverse transcription-quantitative PCR. (B) Concentrations of CCL15 HUVEC culture supernatant in each group were measured by ELISA. (C) Migrated CFs were stained and imaged under a microscope (C-a) Control group, (C-b) NC group and (C-c) Si-CCL15 group. $\mathrm{n}=3$. Scale bar, $100 \mu \mathrm{m}$. (D) The number of migrated CFs in each group. Untransfected HUVECs were used in Ctr groups and non-sense siRNA transfected HUVECs were used in the NC groups. ${ }^{* * *} \mathrm{P}<0.001$ and ${ }^{* * * *} \mathrm{P}<0.0001$ vs. NC; ns vs. Ctr. HUVEC, human umbilical vein endothelial cell; CFs, circulating fibrocytes; Ctr, control; NC, negative control; si, small interfering; CCL, chemokine ligand; ns, non-significant. 

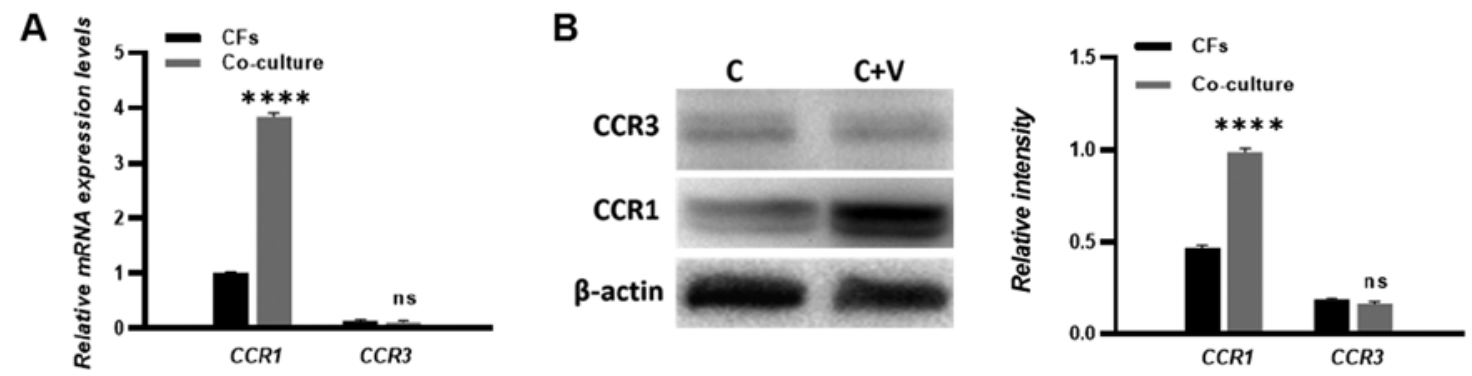

Figure 4. Expression of CCR1 and CCR3 in CFs. (A) mRNA and (B) protein expression levels of CCR1 and CCR3 in the CFs with or without co-culture with human umbilical vein endothelial cells. ${ }^{* * * *} \mathrm{P}<0.0001$ vs. the $\mathrm{CFs}$. CFs, circulating fibrocytes; CCR, chemokine receptor; ns, non-significant.

the CCL15-CCR1 axis, the expression of homologous CCL15 receptors CCR1 and CCR3 was examined in CFs. RT-qPCR and western blotting were performed to measure the expression levels of CCR1 and CCR3. The results showed that the expression of CCR1 was increased in the co-culture compared with the CF single culture. Meanwhile, the expression of CCR3 could not be detected in either the single culture CFs or the HUVECs co-cultured CFs, which suggests a deficiency in CCR3 expression in the CFs (Fig. 4A and B).

To verify if the CCL15-induced CF transmigration was due to interactions between CCL15 and CCR1, CCR1 expression levels were then manipulated. Transfection efficiency of the CCR1 overexpression vector was examined by RT-qPCR and western blotting. The results demonstrated that the expression levels of CCR 1 mRNA and proteins were significantly increased in CFs in the CCR1 overexpression group compared with those in $\mathrm{CF}$ in $\mathrm{NC}$ groups (Fig. 5A and B). CCR1 overexpression in $\mathrm{CFs}$ significantly promoted the migration of $\mathrm{CFs}$ towards HUVECs in co-culture conditions (Fig. 5C and D).

The transfection efficiency of CCR1 shRNA in CFs was examined by RT-qPCR and western blotting. The results demonstrated that the expression levels of CCR 1 in CFs were significantly decreased in the sh-CCR1 group compared with those in $\mathrm{CFs}$ in $\mathrm{NC}$ groups (Fig. 6A and B). In addition, knocking down CCR1 expression significantly attenuated the migration of CFs towards HUVECs in co-culture conditions (Fig. 6C and D).

\section{Discussion}

Chemokines involved in the transmigration of fibrocytes in fibrotic diseases have been widely studied $(8,17,18)$. However, chemokines that regulate the transmigration of fibrocytes during wound healing remain poorly understood. In the present study, chemokines secreted by HUVECs were screened using a gene chip, following which changes in their expression with or without CFs co-culture were compared. To the best of our knowledge, the present study was the first to report that endothelial cell-derived CCL15 modulated the migration of CFs toward HUVECs. CCR 1 and CCR3 are two homologous receptors for CCL15. Notably, the present study demonstrated that CFs only expressed CCR 1 when co-cultured with HUVECs. In addition, the present study demonstrated that overexpression of CCL15 in HUVECs or overexpression of CCR 1 in $\mathrm{CFs}$ promoted the transmigration of CFs towards HUVECs, whilst knockdown of CCL15 or
CCR1 attenuated the migration of CFs towards HUVECs. These results suggest that the CCL15-CCR1 axis serves a vital role in mediating the chemoattraction of CFs towards endothelial cells in vitro.

The use of permeable Transwell co-culture systems to explore the interactions between two cell types are widely used $(19,20)$. The present study co-cultured CFs and HUVECs to mimic the angiogenesis process during wound healing and measured the expression levels of chemokines in HUVECs with or without co-culture with CFs. Gene chip analysis performed in the present study demonstrated that the chemokines CCL2, CCL15 and CXCL8 exhibited the largest fold-changes in expression. Therefore, the effects of CCL2, CCL15 and CXCL8 on CF migration were compared using transmigration assays. The results revealed a potentially vital role for CCL15 in regulating the migration of CFs.

A previous study identified that CCL15 can stimulate chemotactic endothelial cell migration and differentiation, which confirmed the in vitro and in vivo angiogenic activity of CCL15 (21). CCL15 has also been observed to increase the adhesion of human monocytes to endothelial cells under static and shear stress conditions (22). Results from the present study suggested that endothelial cell-derived CCL15 serves an important role in modulating the transmigration of CFs, which is an additional function of CCL15. The results of the present study suggested that the chemokine CCL15 may participate in the angiogenesis process by promoting CF migration, which are the precursors of pericytes (11). Furthermore, the present study also confirmed the expression of CCR1 in CFs. The CCL15-CCR1 axis between endothelial cells and fibrocytes is important. A previous study reported that the CCL15-CCR1 interaction forms a complex tumor-promoting inflammatory microenvironment in human hepatocellular carcinoma (23). Unfortunately, in the present study, the mechanism downstream of the CCL15-CCR1 axis was not explored further, which may have other functions in addition to modulating $\mathrm{CF}$ migration. Therefore, future studies should focus on exploring the CCL15-CCR1 axis in mediating the differentiation of fibrocytes and the activation of downstream signaling pathways related to other functions of fibrocytes.

Chemokines that modulate the transmigration of CFs have been previously explored. CCL2 has been previously found to mediate fibrocyte migration to the lung during asthma, whilst CX3CL1 and CXCL2 can also regulate the transmigration of 
A

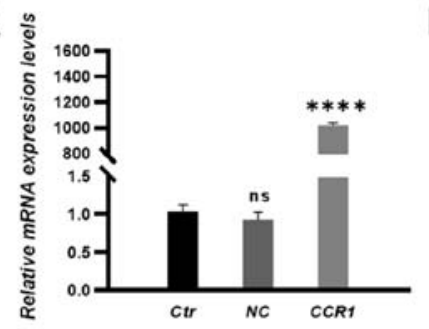

C

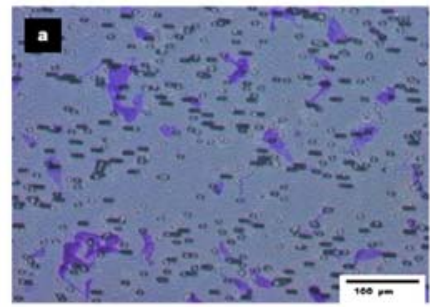

D

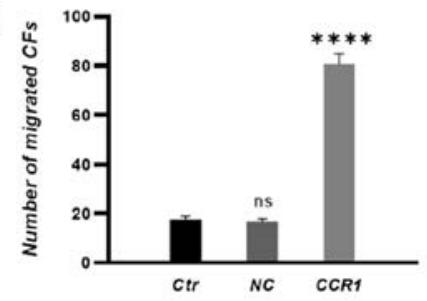

B
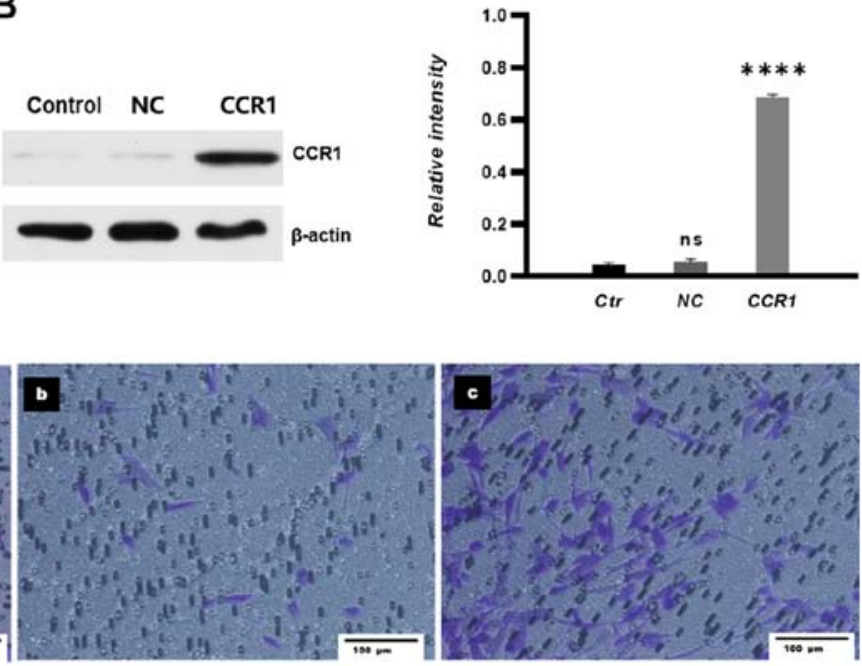

Figure 5. CCR1 overexpression in CFs promotes the migration of CFs towards human umbilical vein endothelial cells in vitro. (A) mRNA and (B) protein expression levels in CFs in each group were measured by reverse transcription-quantitative PCR and western blotting, respectively. Overexpression of CCR1 in CFs was achieved by transfecting them with lentiviral vectors encoding the CCR1 gene. (C) Migrated CFs were stained imaged under a microscope. (C-a) Control group, (C-b) NC group and (C-c) CCR1 group. $\mathrm{n}=3$. Scale bar, $100 \mu \mathrm{m}$. (D) The number of migrated CFs. Untransfected CFs were used in the Ctr groups and empty vector transfected $\mathrm{CFs}$ were used in the NC groups. ${ }^{* * * *} \mathrm{P}<0.0001$ vs. the NC; ns vs. Ctr. CFs, circulating fibrocytes; $\mathrm{Ctr}$, control; $\mathrm{NC}$, negative control; CCR, chemokine receptor; ns, not-significant.

A

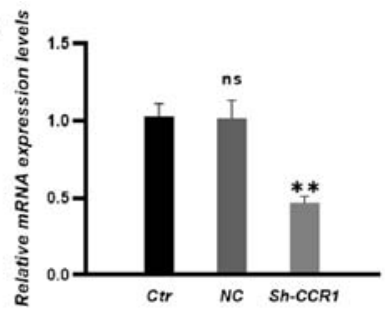

C
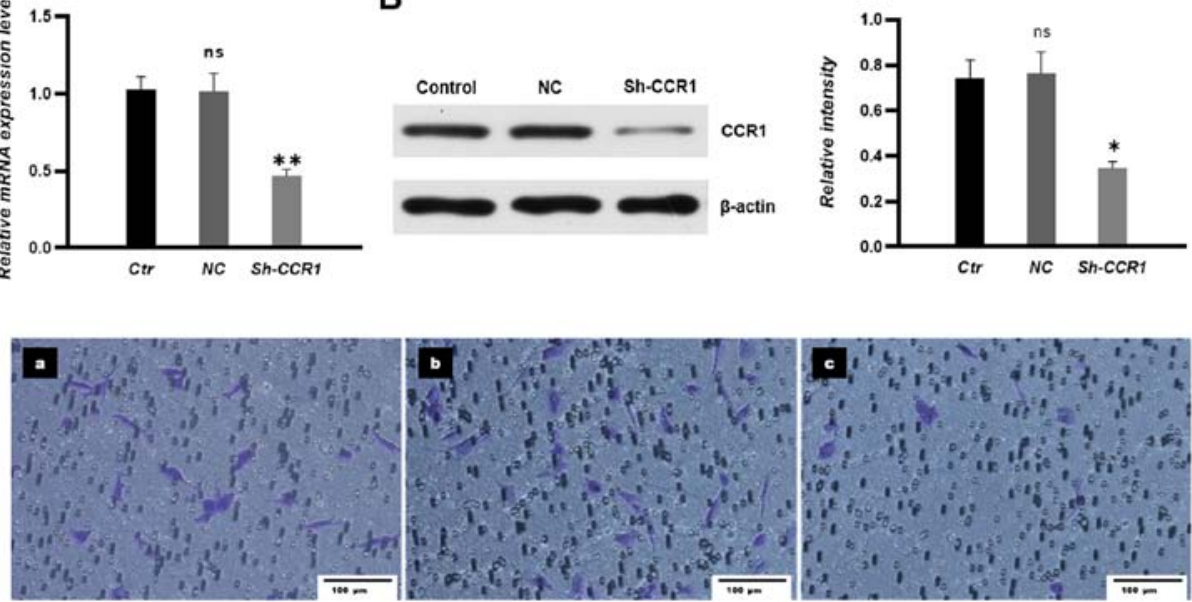

D

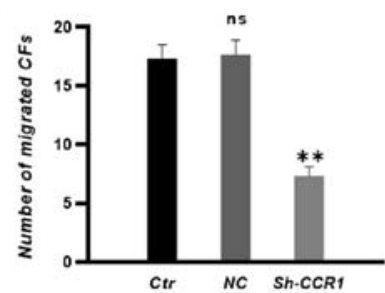

Figure 6. CCR1 knockdown in CFs attenuates the migration of CFs towards human umbilical vein endothelial cells in vitro. (A) mRNA and (B) protein expression levels in each group were measured by reverse transcription-quantitative PCR and western blotting, respectively. (C) Migrated CFs were stained and imaged under a microscope. (C-a) Control group, (C-b) NC group and (C-c) short hairpin-CCR1 group. n=3. Scale bar, $100 \mu \mathrm{m}$. (D) The number of migrated CFs. Untransfected CFs were used in Ctr groups and non-sense shRNA transfected CFs were used in the NC groups. ${ }^{*} \mathrm{P}<0.05,{ }^{* *} \mathrm{P}<0.01$ vs. NC; ns vs. Ctrl. CFs, circulating fibrocytes; Ctr, control; NC, negative control; sh, short hairpin; CCR, chemokine receptor; ns, non-significant. 
CFs $(18,24)$. In the present study, although gene chip resulted in the identification of several chemokines a limitation of the present study is that only three chemokines were selected based on their fold-changes in expression. Therefore, the roles of other chemokines identified by gene chip analysis in mediating fibrocyte migration during angiogenesis may have been overlooked. In addition to transmigration, differentiation of fibrocytes into pericytes is equally vital in the process of angiogenesis (24). However, the present study did not examine the differentiation ratio in fibrocytes after co-culture with HUVECs. Whether the CCL15-CCR1 axis is involved in CF differentiation of CFs require further study.

In conclusion, to the best of our knowledge, the present study identified an important role of CCL15 in mediating the recruitment of fibrocytes by endothelial cells for the first time. The present study also confirmed the expression of CCR1 in fibrocytes and determined the regulatory role of the CCL15-CCR1 axis during the recruitment process of circulating fibrocytes.

\section{Acknowledgements}

The authors would like to thank Professor Xueyong Li (Department of Plastic Surgery, Tangdu Hospital, Fourth Military Medical University) for guiding the present research.

\section{Funding}

This study was supported by the National High Technology Research and Development Program of China (863 Program; grant no. SS2015AA020313) and the National Nature Science Foundation of China (grant no. 81701909).

\section{Availability of data and materials}

The datasets used and/or analyzed during the current study are available from the corresponding author on reasonable request.

\section{Authors' contributions}

ZL and NP performed the experiments and wrote the manuscript. XW and LX analyzed the data and revised the manuscript. RH and XX performed the experiments. XuL conceived and designed the study, prepared the figures and revised the manuscript. JL and XiL analyzed the data. All authors read and approved the final manuscript.

\section{Ethics approval and consent to participate}

This study was approved by the Ethics Committee for Experimentation of the Fourth Military Medical University (approval no. TDLL-20170129; Jan 2017).

\section{Patient consent for publication}

Not applicable.

\section{Competing interests}

The authors declare that they have no competing interests.

\section{References}

1. Cho H, Blatchley MR, Duh EJ and Gerecht S: Acellular and cellular approaches to improve diabetic wound healing. Adv Drug Deliver Rev 146: 267-288, 2019.

2. Jones RE, Foster DS and Longaker MT: Management of chronic wounds-2018. JAMA 320: 1481-1482, 2018.

3. Bucala R, Spiegel LA, Chesney J, Hogan M and Cerami A: Circulating fibrocytes define a new leukocyte subpopulation that mediates tissue repair. Mol Med 1: 71-81, 1994.

4. Chen D, Zhao Y, Li Z, Shou K, Zheng X, Li P, Qi B and Yu A: Circulating fibrocyte mobilization in negative pressure wound therapy. J Cell Mol Med 21: 1513-1522, 2017.

5. Li J, Tan H, Wang X, Li Y, Samuelson L, Li X, Cui C and Gerber DA: Circulating fibrocytes stabilize blood vessels during angiogenesis in a paracrine manner. Am J Pathol 184: 556-571, 2014.

6. Hartlapp I, Abe R, Saeed RW, Peng T, Voelter W, Bucala R and Metz CN: Fibrocytes induce an angiogenic phenotype in cultured endothelial cells and promote angiogenesis in vivo. FASEB J 15: 2215-2224, 2001.

7. Chen Z, Meng P, Li HT, Li M, Yang LF, Yan Y, Li YT, Zou XL, Wang DY and Zhang TT: Thymic stromal lymphopoietin contribution to the recruitment of circulating fibrocytes to the lung in a mouse model of chronic allergic asthma. J Asthma 55: 975-983, 2018.

8. Isgrò M, Bianchetti L, Marini MA, Bellini A, Schmidt M and Mattoli S: The C-C motif chemokine ligands CCL5, CCL11, and CCL24 induce the migration of circulating fibrocytes from patients with severe asthma. Mucosal Immunol 6: 718-727, 2013.

9. Liu Y, Qingjuan S, Gao Z, Deng C, Wang Y and Guo C: Circulating fibrocytes are involved in inflammation and leukocyte trafficking in neonates with necrotizing enterocolitis. Medicine (Baltimore) 96: e7400, 2017.

10. Aono Y, Kishi M, Yokota Y, Azuma M, Kinoshita K, Takezaki A, Sato S, Kawano h, Kishi J, Goto h, et al: Role of platelet-derived growth factor/platelet-derived growth factor receptor axis in the trafficking of circulating fibrocytes in pulmonary fibrosis. Am J Resp Cell Mol 51: 793-801, 2014.

11. Xueyong L, Shaozong C, Wangzhou L, Yuejun L, Xiaoxing L, Jing L, Yanli W and Jinqing L: Differentiation of the pericyte in wound healing: The precursor, the process, and the role of the vascular endothelial cell. Wound Repair Regen 16: 346-355, 2008.

12. Livak KJ and Schmittgen TD: Analysis of relative gene expression data using real-time quantitative PCR and the 2(-Delta Delta C(T)) method. Methods 25: 402-408, 2001.

13. Sambrook J and Russell DW: Calcium-phosphate-mediated transfection of cells with high-molecular-weight genomic DNA. Cold Spring Harbor Protoc 2006: pdb.prot3872, 2006.

14. Smadja DM, Dorfmüller P, Guerin CL, Bieche I, Badoual C, Boscolo E, Kambouchner M, Cazes A, Mercier O, Humbert M, et al: Cooperation between human fibrocytes and endothelial colony-forming cells increases angiogenesis via the CXCR4 pathway. Thromb Haemostasis 112: 1002-1013, 2014.

15. Yamamoto T, Kawada K, Itatani Y, Inamoto S, Okamura R, Iwamoto M, Miyamoto E, Chen-Yoshikawa TF, Hirai h, Hasegawa S, et al: Loss of SMAD4 promotes lung metastasis of colorectal cancer by accumulation of $\mathrm{CCR}^{+}$Tumor-associated neutrophils through CCL15-CCR1 axis. Clin Cancer Res 23: 833-844, 2017.

16. Inamoto S, Itatani Y, Yamamoto T, Minamiguchi S, Hirai h, Iwamoto M, Hasegawa S, Taketo mM, Sakai Y and Kawada K: Loss of SMAD4 promotes colorectal cancer progression by accumulation of myeloid-derived suppressor cells through the CCL15-CCR1 chemokine axis. Clin Cancer Res 22: 492-501, 2016.

17. Singh SR, Sutcliffe A, Kaur D, Gupta S, Desai D, Saunders R and Brightling CE: CCL2 release by airway smooth muscle is increased in asthma and promotes fibrocyte migration. Allergy 69: 1189-1197, 2014.

18. Ishida Y, Kimura A, Nosaka M, Kuninaka Y, Hemmi h, Sasaki I, Kaisho T, Mukaida N and Kondo T: Essential involvement of the CX3CL1-CX3CR1 axis in bleomycin-induced pulmonary fibrosis via regulation of fibrocyte and M2 macrophage migration. Sci Rep 7: 16833, 2017.

19. Bian Y, Du Y, Wang R, Chen N, Du X, Wang Y and Yuan H: A comparative study of HAMSCs/HBMSCs transwell and mixed coculture systems. IUBMB Life 71: 1048-1055, 2019. 
20. Zhang C, Barrios MP, Alani RM, Cabodi M and Wong JY: A microfluidic transwell to study chemotaxis. Exp Cell Res 342: 159-165, 2016.

21. Hwang J, Kim CW, Son KN, Han KY, Lee KH, Kleinman HK, Ko J, Na DS, Kwon BS, Gho YS and Kim J: Angiogenic activity of human CC chemokine CCL15 in vitro and in vivo. FEBS Lett 570: 47-51, 2004

22. Park KH, Lee TH, Kim CW and Kim J: Enhancement of CCL15 expression and monocyte adhesion to endothelial cells (ECs) after hypoxia/reoxygenation and induction of ICAM-1 expression by CCL15 via the JAK2/STAT3 pathway in ECs. J Immunol 190: 6550-6558, 2013.
23. Liu LZ, Zhang Z, Zheng BH, Shi Y, Duan M, Ma LJ, Wang ZC, Dong LQ, Dong PP, Shi JY, et al: CCL15 recruits suppressive monocytes to facilitate immune escape and disease progression in hepatocellular carcinoma. Hepatology 69: 143-159, 2019.

24. Harris DA: Inhibiting CXCL12 blocks fibrocyte migration and differentiation and attenuates bronchiolitis obliterans in a murine heterotopic tracheal transplant model. J Thoracic Cardiovasc Surg 145: 854-861, 2013.

cC) (i) $\odot$ This work is licensed under a Creative Commons cc) International (CC BY-NC-ND 4.0) License. 